\title{
DESARROLLO SOCIAL, POLÍTICAS PÚBLICAS Y CARRERA DOCENTE EN LA REPÚBLICA ARGENTINA. PRESENTE Y FUTURO DE LA FORMACIÓN DOCENTE CONTINUA
}

\author{
SOCIAL DEVELOPMENT, PUBLIC POLICIES AND TEACHING \\ CAREER IN THE ARGENTINE REPUBLIC. PRESENT AND FUTURE \\ OF CONTINUOUS TEACHER TRAINING
}

\author{
Daniel Ernesto Stigliano
}

\begin{abstract}
Argentina undertook in 2007 an important educational reform with the enactment of the National Education Law. However, eleven years after the beginning of this innovative process and eight years after the publication of the OEI 2021 Educational Goals, no significant quality improvements are evident from the different assessments that are administered annually by different international organizations. The initial and continuous teacher training is an indispensable pillar for the sustainable development of a society. Educational policies for innovation are successful when they manage to act simultaneously on the rules and subjectivity of teachers. The political neglect of the second variable has generated "renewed positions occupied by traditional teachers". This paper analyzes the current moment of continuous teacher training in Argentina and lists some proposals for improving the teaching career and its impact on the learning process of students.
\end{abstract}

Key words: Teachers Training and Social Development, Law 26206, Trajectory in the management, Trajectory in the classroom

\section{RESUMEN}

La Argentina emprendió en el 2007 una importante reforma educativa con la sanción de la Ley de Educación Nacional. Sin embargo, a once años del comienzo de este proceso innovador y a ocho años de la publicación de las Metas Educativas 2021 de la OEI no se evidencian mejoras de calidad significativas a partir de las distintas evaluaciones que administran anualmente diferentes organismos internacionales. La formación docente inicial y continua es un pilar indispensable para el desarrollo sustentable de una sociedad. Las políticas educativas para la innovación resultan exitosas cuando logran accionar simultáneamente sobre la normativa y la subjetividad de los docentes. La desatención política de la segunda variable ha generado puestos renovados ocupados por docentes tradicionales. En este trabajo se analiza el momento actual de la formación docente continua en la Argentina y se enumeran algunas propuestas para la mejora de la carrera docente y su impacto en el proceso de aprendizaje de los estudiantes.

Palabras clave: Formación Docente y Desarrollo Social, Ley 26206, Trayectoria Directiva, Trayectoria Aúlica

Fecha de recepción: 16 de noviembre de 2018

Fecha de aceptación: 18 de enero de 2019 


\section{INTRODUCCIÓN}

Un estudio publicado por el Instituto de Estadística de la UNESCO (IEU) y el Informe de Seguimiento de la Educación para Todos en el Mundo revelan que se está incrementando el número de niños y adolescentes sin escolarizar, una cifra que en 2013 ascendió a 124 millones. El estudio muestra que la ayuda internacional para la educación sigue por debajo de los niveles de 2010 y resulta claramente insuficiente para alcanzar las nuevas metas relativas a la universalización de la enseñanza primaria y secundaria.

Los nuevos datos del IEU muestran que 1 de cada 11 niños está sin escolarizar, lo que en 2013 sumaba un total de 59 millones de niños. Es decir: 2,4 millones más que en 2010. De ellos, 30 millones viven en el África Subsahariana y 10 millones en el Asia Meridional y Occidental. Según los cálculos del IEU, 24 millones de niños nunca pisarán un aula. En el África Subsahariana, la mitad de los niños sin escolarizar no llegará nunca a matricularse. Las niñas son las más desfavorecidas, especialmente en el Asia Meridional y Occidental, donde el 80\% sin escolarizar tiene escasas probabilidades de empezar a ir a la escuela, frente al 16\% de los niños. Además, 1 de cada 6 adolescentes no está escolarizado, lo que suponía un total de 65 millones en 2013. Una tercera parte de ellos vive en el Asia Meridional y Occidental y otra tercera parte en el África Subsahariana, donde hoy hay más adolescentes sin escolarizar que en 2000.

Otro factor que incide negativamente sobre el analfabetismo, la repitencia y la deserción escolar son los conflictos internos que provocan crisis de desplazamiento de miles de migrantes que buscan una vida mejor lejos de los enfrentamientos civiles y el hambre. Estos procesos se han manifestado regionalmente en toda América Latina con un crecimiento constante en los últimos años. Las corrientes migratorias han provocado situaciones novedosas que todavía los diferentes sistemas educativos nacionales no terminan de comprender. Estos efectos pertenecen tanto a la dimensión estructural, que no está preparada para ofrecer el número de vacantes que solicitan los migrantes, como a la dimensión educativa que debe prestar atención al tratamiento de la diversidad cultural. Estos fenómenos no se pueden ignorar ya que también requerirán de docentes con una experticia profesional muy específica para educar en esta nueva realidad.

Un estudio del Proyecto Mitigación de la Pobreza y Desarrollo Social del Programa de las Naciones Unidas para el Desarrollo (PNUD) sostiene que "la educación es una inversión cuya tasa de retorno es relativamente alta comparada con la de otros proyectos". También asegura que "las tasas de retorno de la educación en países en desarrollo son más altas que en países ya desarrollados, lo que sugiere que existen importantes oportunidades de inversión aún no aprovechadas". Finalmente, afirma que "la tasa de retorno de la educación primaria tiende a superar a la obtenida en los niveles secundarios y terciarios, particularmente en países menos desarrollados" (Larrañaga, 2014, p.6).

Este análisis revela que la educación no es un gasto sino una inversión y que los recursos en muchas ocasiones se invierten mal. De acuerdo con estas conclusiones del estudio, las políticas de Estado a mediano plazo deberían contemplar una inversión en la educación inicial, con un crecimiento paulatino a lo largo de diez o quince años, hasta abarcar todos los ciclos de escolarización (primaria y secundaria) para favorecer el desarrollo integral de sus comunidades.

En particular, la problemática de la escuela secundaria configura en la Argentina y en otros países de Latinoamérica, una preocupación importante para quienes tienen la responsabilidad de tomar decisiones políticas para el sector, como así también para los investigadores y los educadores que 
despliegan su trabajo en la escuela secundaria. "La escuela secundaria constituye uno de los niveles del sistema educativo que genera mayores controversias en cuanto a la necesidad de su modificación" (Ziegler, 2001, p.3). En el presente trabajo se relacionarán una serie de dificultades que atraviesa la educación media argentina con las actuales propuestas del sistema para la formación de los profesores en ejercicio de la cátedra. Para ello, se tomarán como referencia algunas consideraciones del Documento Final de las Metas Educativas 2021 de la OEI A partir de esa discusión se enumerarán una serie de propuestas para la mejora de la capacitación docente continua y la carrera docente.

\section{LAS METAS EDUCATIVAS EN LA ARGENTINA}

El 14 de diciembre del año 2006 se sancionó la Ley de Educación Nacional Nº 22206 (LEN) que estableció la obligatoriedad de la escuela secundaria (Art. 16) en todo el territorio nacional. La mencionada norma, tiene por objeto regular el ejercicio del derecho de enseñar y aprender consagrado por el artículo 14 de la Constitución Nacional y los tratados internacionales incorporados a ella. En su articulado, establece la estructura del Sistema Educativo Nacional haciendo alusión a todos los niveles y modalidades de la enseñanza. Determina la extensión de la obligatoriedad escolar y plantea las responsabilidades y obligaciones del Estado Nacional, las Provincias y la Ciudad Autónoma de Buenos Aires en relación a la Educación. Desde su puesta en vigencia se aplica en las 24 jurisdicciones con las que cuenta el sistema, respetando los criterios federales, las diversidades regionales y articula la educación formal y no formal, la formación general y la profesional en el marco de la educación continua y permanente.

La LEN establece 14 años de escolarización obligatoria distribuidos en dos años para la educación inicial (salas de 4 y 5 años), siete para la educación primaria y cinco para la secundaria.

Así mismo, la Educación Secundaria se divide en dos ciclos: un Ciclo Básico, de carácter común a todas las orientaciones y un Ciclo Orientado, de carácter diversificado según distintas áreas del conocimiento, del mundo social y del trabajo.

Para hacer efectivo el cumplimiento de la obligatoriedad, como un derecho de niños y jóvenes, se requiere la puesta en marcha de una diversidad de acciones y estrategias que posibiliten una inclusión educativa genuina. Entre ellas, se destaca la introducción de ciertas innovaciones organizacionales y pedagógicas que posibiliten a los adolescentes y jóvenes la realización de una experiencia escolar significativa en las instituciones de educación secundaria. La generación de propuestas de escolarización tendientes al cumplimiento de la obligatoriedad escolar, requiere la modificación de uno o más componentes del denominado formato escolar tradicional, cuestión que pone en tensión el vínculo entre la administración y la escuela.

El Documento Final de las Metas Educativas 2021 de la OEI afirma que:

"Si se piensa en la calidad de la educación de un país, es inevitable hacerlo en relación con la calidad de su profesorado. De ahí la prioridad que la gran mayoría de las reformas educativas otorga al fortalecimiento de la profesión docente. Como pone de manifiesto la investigación comparada, los países que logran los mejores resultados en las evaluaciones internacionales cuidan especialmente a su profesorado: seleccionan a los candidatos a la formación docente en el tercio superior de los egresados de la educación secundaria; ofrecen buenos salarios iniciales para hacer de la docencia un profesión atractiva, y presentan múltiples oportunidades de mejora durante la carrera profesional" (OEI, 2010, p.74) 
Ni con la mejor ley de educación se consigue transformar la estructura de un sistema educativo. El cambio no se produce si no se trabaja el modelo educativo con los educadores. De allí que los sistemas de formación docente son fundamentales para asegurar la calidad educativa de una nación y su desarrollo humano y económico.

"Un profesor que cuente con una formación inicial de calidad y con las oportunidades de acceder a programas de capacitación continua, puede contribuir al mejoramiento de los resultados de los niños en su rendimiento. Sin embargo, en la mayor parte de los países de la región, las inmensas demandas de transformación pedagógica que se les ha exigido a los docentes en las últimas décadas no han sido acompañadas de los debidos procesos de cambio de las instituciones que los forman, ni de las condiciones de trabajo y de desarrollo profesional necesarias" (OEI, 2010, p.74)

Estos procesos no son desconocidos para el sistema educativo argentino. Hace quince años, en un contexto político diferente Emilio Tenti Fanfani (1999) planteaba dos paradojas que aún siguen presentes a pesar de los pasos andados en los años posteriores: "nuevos maestros en puestos viejos", "puestos renovados ocupados por individuos tradicionales" (p.99).

¿Cuándo ocurre la primera situación? Toda vez que se producen transformaciones en la subjetividad de los profesores como resultado de las grandes reformas educativas (como la que nos ocupa desde el 2007) sin que se reestructuren las reglas y recompensas del puesto de trabajo el resultado es un malestar que se manifiesta en el colectivo de los docentes. Este fenómeno se puede resolver de dos maneras, "o bien el puesto termina por doblegar al ocupante o bien los ocupantes, mediante estrategias colectivas adecuadas, logran desestructurar y reestructurar el sistema de reglas que definen la posición de los maestros". El citado investigador señala que las reformas institucionales "vienen después de la transformación de los agentes". (Tenti Fanfani, 1999, p.100)

Esta transformación de los agentes supondrá enfrentar los miedos a perder conocimientos emblemáticos o núcleos tradicionales, a enseñar contenidos para los que no se está preparado, a bajar los niveles de aprendizaje, a que los alumnos aprendan menos y por sobre todo miedo a la pérdida de identidad. "Modificar la realidad forma parte del Yo, pero a condición de que se recuerde qué se quiere modificar. La modificación no destruye lo anterior. Modificar el granero para convertirlo en biblioteca no es destruirlo, es respetar sus características, pero cambiarlas para hacerlo más aprovechable o habitable". (Aulagnier, 1976, p.12).

¿Cuándo tiene lugar la segunda posibilidad? Cuando se establecen reformas curriculares, de acceso al puesto o reglamentarias sin considerar que el colectivo docente no se encuentra sociológicamente adaptado para llevar a la práctica las nuevas legislaciones. Este problema ha sido el más frecuente en nuestro país y en América Latina toda vez que se ensayaron reformas educativas o cambios significativos en el puesto de maestro. El resultado es conocido: "las tradiciones que sobreviven bajo la forma incorporada en la subjetividad de los maestros terminan neutralizando lo que está escrito en las leyes, los reglamentos, los planes de estudio y los contenidos" (Tenti Fanfani, 1999, p.100). Como resultado, es que la historia y las creencias implícitas de los profesores sigue imponiendo su determinación sobre el presente a través de su impacto sobre los modos de enseñar y de querer (o de hacer las cosas) en el aula y en la escuela.

\section{SITUACIÓN ACTUAL DE LA FORMACIÓN DOCENTE CONTINUA}


"El perfeccionamiento y la capacitación docente no pueden plantearse sino en relación con la redefinición de un concepto de profesión." (Huberman 1999, p.25). Desde el punto de vista clásico, se entiende a la capacitación como un "proceso consciente, deliberado, participativo y permanente implementado por un sistema educativo o una organización, con el objetivo de mejorar desempeños y resultados; estimular el desarrollo para la renovación en campos académicos, profesionales o laborales y reforzar el espíritu de compromiso de cada persona para con la sociedad y particularmente para con la comunidad en la cual se desenvuelve." (Huberman 1999, p. 29).

Desde nuestro punto de vista, la capacitación de los docentes se entiende de manera más amplia y "como parte precisa o imprecisa, formal o informal, deliberada o no, del proceso de formación que desarrolla cada persona dentro de su ámbito académico o laboral.” (Huberman 1999, p.31) razón por la cual, esta óptica la capacitación, más que una moda, una carrera de créditos, puntos y premios, necesita ser un objeto de conocimiento, que debe ser investigado en forma continua.

Regresemos al estado actual de la escuela secundaria con palabras de Silvia Kravetz (2012, p.4): "No obstante todas estas iniciativas y los esfuerzos orientados en tal sentido, el sistema educativo argentino aún exhibe la persistencia de viejos problemas a los que se han sumado recientemente nuevos desafíos. Entre los primeros se destacan la repitencia de los primeros años de cada nivel, el abandono temprano en el nivel medio, los logros de aprendizaje distantes a los esperados según los datos arrojados por los Operativos Nacionales de Evaluación, las desigualdades que se advierten en las propuestas de enseñanza para distintos sectores sociales, por nombrar sólo algunos".

Para evitar estos fenómenos expulsivos de los jóvenes se hace necesario concebir estrategias específicas que achiquen la brecha entre las culturas institucionales escolares y las culturas de los niños y adolescentes que hoy pueblan las aulas.

Tanto la LEN como las Resoluciones del Consejo Federal de Educación No 83 y 94 que propician una "nueva escuela secundaria" hacen posible la emergencia de ciertas innovaciones institucionales y pedagógicas que se distanciarían en mayor o menor medida de lo que se considera el formato institucional tradicional. Estas nuevas estructuras organizativas sumadas a nuevos modelos de convivencia escolar y de evaluación de los aprendizajes que no están previstos en el ordenamiento actual de la administración educativa "podrían instalar tensiones en el "contexto de la práctica" (Ball, Bowe, et al, 1992). Si bien el artículo mencionado alude al conjunto del sistema educativo y por ende a todos los niveles, es posible suponer que el mayor desafío para su cumplimiento se localiza en la escuela secundaria, el tramo más crítico y cuestionado por la comunidad educativa (padres, docentes y alumnos) y por actores de distintos sectores sociales.” (Kravetz, 2012, p.5)

Terigi (2008) expresa que producir cambios en el formato escolar entraña no sólo cambios pedagógicos; agrega que son numerosos los estudios que señalan que el aparato institucional de administración de la educación, requiere que las decisiones políticas se traduzcan en orientaciones operativas que permitan promover los cambios en la dirección que pretenden las políticas. Una de esas decisiones pasa por la capacitación.

Se entiende que toda capacitación de los docentes en ejercicio de la cátedra que se quiera desarrollar en estos tiempos de cambio acelerado no puede ignorar que la profesión se encuentra en crisis y que para salir de ella se deben atender las transformaciones recientes del oficio de enseñar, a saber:

- La educación es una ocupación en desarrollo cuantitativo permanente (Tenti Fanfani, 1999, 
p.95). Su ritmo acompaña al ritmo de crecimiento del PBI, los cambios en la política y las crisis globales. Se debe capacitar a los docentes para los procesos de masificación, globalización y cambio permanente.

- La profesión docente posee una heterogeneidad creciente. La LEN establece cuatro niveles y ocho modalidades, un subsistema de gestión estatal y otro de gestión privada atomizan la realidad del colectivo. Subsiste una deuda histórica con el cuerpo magisterial: el deterioro de las recompensas materiales obligación del estado y el deterioro de las recompensas materiales reflejado en la desconsideración de la sociedad hacia la profesión. Las Metas Educativas 2021 de la OEI (2010, p.73) afirman que:

"Otro estímulo que puede incidir positivamente en el quehacer educativo es el mejoramiento de las condiciones laborales. Al respecto, el aumento de jornada escolar completa puede significar un cambio importante en la vida profesional, ya que permite concentrar el trabajo en un solo establecimiento y no en varios (posibilitando no tener que complementar los ingresos con otras ocupaciones), de manera tal que puede fortalecer la identificación con su proyecto educativo"

- La introducción desigual en la educación de las tecnologías de la información y la comunicación en los modos de hacer que las cosas que generan un impacto que necesita ser atendido. La metamorfosis de la familia (Romero, 2009, p.36). Si la familia nuclear moderna era, en palabras de Elkind (2003), "el tibio refugio de un mundo desalmado", la familia permeable posmoderna es una "bulliciosa estación de trenes donde descansar un rato antes de abordar el próximo tren”. Las Metas educativas 2021 de la OEI (2010) también manifiestan su preocupación por este tema:

"Teniendo en cuenta el escenario en el que se sitúa, para la labor docente implica un gran desafío marcar efectivamente una diferencia respecto a la formación que los niños reciben en su hogar y otros espacios. Los docentes son por antonomasia el cuerpo capacitado para ejercer con legitimidad la función educadora; en ese sentido, se espera de ellos un buen desempeño de sus competencias. Sin embargo, para asegurar aquello no es suficiente la legitimación social que sobre ellos recae: también es necesaria una acreditación oficial de su propia formación como educador. La formación inicial de los docentes se constituye, entonces, como un proceso de vital importancia para las definiciones de una educación de calidad". (p.74)

- La llegada de las TICs y la metamorfosis familiar dan paso también al fenómeno de los "niños competentes y los adolescentes mundanos" (Romero, 2009, p.38) reflejados en el cine como superchicos en películas como "Mi pobre angelito", "Belleza americana" o "Toy story" que dificulta la comunicación interpersonal con docentes que crecieron como niños jugando a las cartas de cartón y un contrincante niño y no con cartas virtuales y una tableta como desafiante.

\section{LA CARRERA DOCENTE EN EL SISTEMA EDUCATIVO NACIONAL}

El sistema de capacitación docente nació en 1958 con el Estatuto del Docente y sigue vigente: maestros y profesores deben sumar puntos aprobando cursos. Cuantos más cursos, más centésimos se acumulan para conseguir la titularidad (estabilidad en un cargo) o ascender a cargos directivos.

El Sistema Educativo Argentino cuenta con abundante normativa para la formación docente inicial pero carece de una normativa unificada para la formación docente continua que abarque todo el 
territorio de la nación. La ausencia de regulaciones centralizadas del gobierno federal se traduce en una diversidad de modalidades de capacitación docente que determina con autonomía cada una de las veinticuatro provincias argentinas.

Este sistema de ascensos era razonable hace medio siglo cuando los maestros se recibían con título secundario y 17 años de edad. El perfeccionamiento, tenía la misión de mejorar las prácticas docentes en épocas en las que no existía formación de grado o posgrado.

Pero hace tiempo que acumular puntos genera prácticas especulativas, con poco o nulo sentido pedagógico. Entre las distorsiones del sistema puede verse que algunos cursos que demandan poco tiempo y esfuerzo brindan, relativamente, más puntaje que una maestría universitaria. O que los docentes titulares que no quieren ascender y no necesitan los puntos, pasan los últimos quince o veinte años de su carrera sin aumento salarial desde que lograron el máximo de antigüedad y sin agenda de mejora personal ni de las escuelas en las que trabajan. O que los docentes hacen cursos dispersos, sin orientación y sin sistematicidad, aclarando que los hacen por propia voluntad, si tienen tiempo y compromiso con la tarea. Además, la demanda de puntos genera una oferta no siempre de buena calidad y una relevante inflación de puntos que hace que la mayoría de los docentes llega rápidamente al máximo permitido.

"Las soluciones siguen sin ser superadoras: poner topes anuales, aumentar el límite máximo o anular los puntos obtenidos y retocar aspectos parciales del sistema de puntaje docente termina por reforzar su matriz burocrática, lejos del avance educativo. Lo mejor que se podría hacer es cambiarlo y pasar a un modelo con autonomía y excelencia. Aquí algunos caminos que requieren voluntad política de cambio: distinguir docentes en formación de docentes formados, la capacitación no puede depender solamente de las decisiones individuales de los docentes, la idea de que el motor del cambio es la capacitación docente atrasa medio siglo" (Narodowsky, 2016, p.1)

Sin embargo, existen algunas posibilidades normativas que no se pueden dejar de lado si se pretende efectuar una reforma profunda del sistema. Estas posibilidades tienen que ver directamente con el significado de progreso o ascenso en la carrera docente y las dos posibilidades a la que abre puertas la Ley de Educación Nacional (LEN): La trayectoria directiva con suma de puntaje por antigüedad y antecedentes o la trayectoria áulica.

La LEN N 26206 en su Artículo 69, sienta las bases para definir los criterios concernientes a la carrera docente e introduce un concepto novedoso para la tradición histórica del sistema educativo argentino que define como diferenciación de trayectorias. Actualmente, cómo se decía líneas atrás, la única posibilidad de hacer carrera en la docencia, es a través de puestos (maestro/profesor; vicedirector; director; supervisor). Este sistema de ascenso no tiene en cuenta las competencias personales de los docentes y privilegia como instrumento de progreso en el escalafón los años de antigüedad que se acumulan en servicio.

La LEN diferencia la llamada y conocida trayectoria directiva con un concepto novedoso, el de trayectoria aúlica. Con este sistema, el docente podría ascender y mejorar su remuneración sin salir del aula y sin descuidar la tarea para la que se formó: enseñar. En esta instancia "la capacitación se transforma en una de las dimensiones básicas para el ascenso en la carrera profesional" (Artículo 69 de la LEN). 
El sistema propuesto se asemeja al existente en las universidades donde los profesores recorren su carrera profesional sin dejar de impartir clases, atravesando diferentes instancias jerárquicas (ayudante, jefe de trabajos prácticos, profesor adjunto, profesor titular) que no se traducen en un puesto de trabajo como en la trayectoria directiva.

¿Pero con cualquier tipo de capacitación? Los docentes que están en formación son los recién recibidos, que necesitan apoyo por parte de los educadores formados y deberían complementar su primeros pasos en la docencia no con cursos dispersos sino con trayectorias sistemáticas de grado y posgrado universitarios (maestrías, especializaciones). En el país existe una oferta variada y heterogénea, de calidad superior respecto de la mayoría de los cursos de capacitación tradicionales. Por su parte, los docentes formados precisan reconocimiento simbólico y económico que estimule su compromiso con la buena enseñanza.

La capacitación no puede depender solamente de las decisiones individuales de los docentes. Ella debe implementarse en cada escuela, como ya se ha dicho, en función de las necesidades pedagógicas concretas. Los modernos y complejos sistemas escolares no apuestan a la voluntad individual de los docentes sino a escuelas reflexivas, centros de decisión pedagógica. La función del Estado es poner a disposición de las instituciones educativas un menú amplio de opciones de capacitación en servicio que satisfaga la demanda profesional de educadores concretos que tienen que resolver los problemas educativos concretos que son producto de la realidad del contexto sociocultural y que difieren de una institución educativa a otra.

En nuestra época es la innovación la que marca la tendencia y la capacitación debe supeditarse a ella. Porque la mejora educativa no es la sumatoria de habilidades de cada educador sino que se expresa cuando existen condiciones para la innovación, el trabajo en equipo, en red, y la autoevaluación institucional en un contexto profesional emprendedor y no desjerarquizado como el que experimentamos cotidianamente. Por eso, se hace urgente reemplazar al sistema de puntaje por el reconocimiento de estrategias y perfiles pedagógicos innovadores.

\footnotetext{
"Ya es tarde como para seguir rindiéndole culto al tótem del puntaje docente y emparchar este viejo sistema no hace más que consolidarlo. Dejemos de lado cuántos centésimos van al último cursito de capacitación. Cambiemos las condiciones reales de las escuelas (recursos, reglas, incentivos), para así brindar capacidad pedagógica real a los equipos escolares para mejorar las escuelas reales" (Narodowsky, 2016, p.2)
}

\section{DISCUSIONES Y CONCLUSIÓN}

Hasta la fecha, ninguna provincia argentina ha avanzado en la reglamentación jurisdiccional del Artículo 69 de la LEN. Seguidamente se enumerarán algunas propuestas para delinear la trayectoria aúlica en el sistema educativo nacional. Antes de avanzar con ellas se cree oportuno enumerar tres supuestos básicos que no se pueden perder de vista si se desean obtener resultados satisfactorios:

- Cualquier modificación que se introduzca en el sistema e implique la condición laboral y profesional de los docentes debe contar con una verdadera apropiación por parte del colectivo 
de profesores. Sin esta conquista previa lo más probable será encontrar un escenario de resistencia. ¿Cómo lograrlo? A través de mecanismos de consulta que involucren a los docentes y a sus asociaciones sindicales en el proceso de construcción de la nueva normativa ya que como hemos dicho en otro momento de esta investigación "son los actores institucionales los que controlan a las escuelas y no las instituciones escolares a sus actores" (Valsiner, 2005, p.3). En otras palabras, la educación se transforma de abajo hacia arriba. Las Metas Educativas 2021 de la OEI aseguran al respecto: "vemos que otra forma de mejorar el bienestar subjetivo de los docentes y su motivación es elevar el prestigio de su labor. De ese modo, habrá una mayor atracción de los postulantes a la educación superior, y la respuesta de la sociedad se traducirá en un respeto y valoración por su trabajo, cuestión que hoy en día se ha visto fuertemente deteriorada OEI (2010, p.75)

- Ningún cambio profundo se puede implementar en un solo paso, requiere de un plan de aplicación gradual y progresivo. Probablemente, convenga mantener vigentes ambas trayectorias para permitir que cada docente escoja su propio futuro profesional como agente del sistema educativo. Indudablemente, se registrará en un primer momento una opción baja por la trayectoria áulica. En la medida, que los primeros docentes que transiten por ella expresen su satisfacción por la elección, es esperable que un mayor número de docentes se pase del sistema de ascenso tradicional a la trayectoria áulica.

- Para que la trayectoria áulica sea aceptada no sólo se deben ofrecer recompensas simbólicas. Se deberán prever recompensas materiales significativas desde el principio de su implementación.

A continuación se sugieren algunas propuestas alternativas no tradicionales para la capacitación de docentes en servicio y el estímulo de la trayectoria aúlica como por ejemplo tutorías, distinciones y premios, y pasantías.

Tutorías: Instancia de capacitación en servicio en la que se relaciona a un docente con un especialista universitario destacado de la disciplina que él enseña (por ejemplo un investigador científico con un profesor de ciencias naturales). El tutor transmite al tutoreado su experiencia y sabiduría mientras que este incrementa sus conocimientos de base, mejora habilidades existentes y adquiere otras nuevas.

Investigación, distinciones y premios: Estimular a los docentes a adoptar una actitud profesional que incluya una inclinación hacia la investigación, la redacción de trabajos para congresos de la especialidad y la publicación de materiales didácticos y científicos. Para ello, habría que ofrecer el acceso a subsidios para la investigación en colaboración con instituciones de alto reconocimiento social y flexibilizar el régimen laboral. Actualmente, la participación de un docente en un congreso implica agotar las licencias por razones particulares y el descuento automático del rubro salarial conocido como presentismo. Otra mejora, es el otorgamiento de un semestre sabático con goce de haberes destinado a estas actividades con un reglamento estricto que establezca tiempos, criterios para la postulación, presentación de los informes de avance y el producto que los participantes deberán informar al finalizar la experiencia.

Pasantías: Los docentes podrían realizar períodos de pasantías, con relevamiento de tareas, en empresas, laboratorios, museos, bibliotecas, etc. de acuerdo con la formación de base. De este modo, se podría, más allá de la construcción de nuevos saberes profesionales, disminuir la brecha existente entre el saber escolar y el saber erudito. "Por otra parte, los diseños de la Formación 
debieran contemplar también la importancia tanto de las asociaciones y las instituciones superiores y universitarias especializadas como de los movimientos profesionales de base"(Moglia, 2008, p.162)

En cuanto al ingreso a la docencia, se puede seguir contemplando, como hasta el momento dos vías, la titulación docente de base o la titulación en alguna profesión laboral. En este último caso se debería exigir una capacitación pedagógica obligatoria ofrecida por el sistema. Entendemos que es beneficiosa la incorporación a la docencia de profesionales, especialmente en las áreas de ciencia, tecnología y tecnologías de la comunicación, pero del mismo modo que para un docente de carrera especializado en una determinada disciplina no es suficiente saber pedagogía tampoco es suficiente para un profesional que accede a la docencia conocer sólo su disciplina.

Cómo se ha visto a lo largo de este trabajo aún queda mucho por hacer. Si se presta atención al discurso de los actores políticos de nuestro país, escucharemos que afirman que una mejor calidad de la educación se alcanza con una mayor cantidad de días de clase cuando, en realidad, la calidad educativa no se define por el tiempo de permanencia en la escuela, sino por el tipo de actividades que docentes y alumnos desarrollan durante el tiempo de clase. De nada vale una escolaridad de jornada completa ni 190 anuales de clase, si durante el tiempo escolar sólo se desarrollan tareas de contención que no están dirigidas al desarrollo de los diferentes procesos cognitivos, como la creatividad, la resolución de problemas y la metacognición, fundamentales para enfrentar un mundo cambiante y con renovados desafíos. Sorprendentemente, quienes sostienen este discurso nos invitan a "copiar" el modelo educativo finlandés que, con uno de los mejores índices de aprendizaje del globo, cuenta con muchos menos días de clase al año y menos horas de clase diarias que la Argentina.

La mejora pasa indudablemente por otro lado. Sería ingenuo pensar que una escuela tradicional pueda formar ciudadanos preparados para vivir en un futuro incierto. Cabe mencionar el pensamiento de Amartya Sen (1992, p.6) cuando sostiene que "el bienestar de las personas depende de sus capacidades para desarrollar plenamente distintas actividades relacionadas con el quehacer humano". Los niños que este año ingresan a la escuela primaria finalizarán su escolarización en 2030, cuando se encontrarán con oficios y profesiones hoy inexistentes e imposibles de imaginar. No se logrará una educación de calidad si no se trabaja en el tiempo escolar y extraescolar para lo desconocido. Sin embargo, es imposible enseñar y aprender para lo desconocido con aulas de cincuenta estudiantes, escasos recursos didácticos, alumnos que van a la escuela para alimentarse, o sin espacios gratuitos y accesibles para que los educadores puedan capacitarse sobre este nuevo formato escolar que desafía y trasciende los muros.

Regresando a los propósitos de este trabajo se ha establecido la íntima relación que existe entre una educación de calidad para el desarrollo, la capacitación de los docentes responsables de administrarla y una carrera docente con estímulos adecuados. Cuando se hace referencia a una educación de calidad, se lo hace pensando en la escuela más cara o en la de mejor infraestructura y equipamiento, sino en una escuela donde los alumnos aprendan. Una educación de calidad es, ni más ni menos, aquella en la que los alumnos aprenden.

Finalmente se considera pertinente sumar estas propuestas:

- Operar simultáneamente sobre la normativa educativa que, por su naturaleza burocrática, en general se opone a los procesos de innovación y sobre los docentes y equipos de conducción, 
para consensuar cambios sustanciales en los modos de enseñar y aprender para lo desconocido. No se debe perder de vista que la educación se transforma de abajo hacia arriba (desde las escuelas) y no por las normas que la reglamentan. Un país puede contar con la mejor Ley de Educación, pero si no cambia lo que se hace adentro de las aulas es imposible que logre por arte de magia lo que está impreso en unas hojas de papel.

- Involucrar a las familias, a la academia, a las entidades sindicales y a las organizaciones no gubernamentales en un proceso que cultive la cultura del diálogo y el encuentro para la mejora de la educación.

- Generar redes de cooperación entre docentes, organizaciones vinculadas a la educación y universidades, con el objeto de encontrar soluciones locales para los diferentes problemas que obstaculizan el aprendizaje intelectual y emocional de los niños y los jóvenes.

- Trabajar por una inclusión verdadera. Toda la producción de normativa adopta actualmente el discurso de la inclusión, pero paradójicamente no se facilitan las condiciones para que se pueda instrumentar en la práctica. Para entender la inclusión, primero hay que comprender otros dos conceptos que, en general, se confunden, pero son muy diferentes: igualdad y equidad. Mientras que la igualdad consiste en respetar los mismos derechos para todos, la equidad consiste en dar a cada uno lo que necesita. A modo de ejemplo, si las autoridades nacionales entregan gratuitamente ordenadores personales a todos los alumnos escolarizados, incluidos los de zonas rurales donde no existen posibilidades de conexión a la web o no hay energía eléctrica, se trata de una política que busca la igualdad pero no la equidad. No existe inclusión sin equidad.

Se vuelve a afirmar que los tres conceptos que puso en diálogo este trabajo: desarrollo social, políticas para la educación y formación docente son tres conceptos que van de la mano y no pueden interactuar por separado sin mirarse uno al otro. Ante la urgencia de la inequidad se hace prioritario intensificar la innovación y el cambio en la formación docente sin perder de vista que una educación con buenos índices de calidad se consigue con maestros y profesores de excelente formación y con escuelas que además de cumplir un rol de contención social ante los problemas estructurales de las economías regionales permitan que los alumnos aprendan. 


\section{BIBLIOGRAFÍA}

Aulagnier, P. (1976) La violencia de la interpretación. Buenos Aires: Amorrortu.

Elkind D. (2003) La escolaridad y la familia en el mundo posmoderno. En Hargreaves, A.(comp.) Replantear el cambio educativo. Un enfoque renovador. Buenos Aires: Amorrortu

Huberman S. (1999) Como se forman los capacitadores. Artes y saberes de su profesión. Buenos Aires: Paidos.

Kravetz, S. (2012) Formato escolar y obligatoriedad de la escuela secundaria. Reflexiones desde la perspectiva del ciclo de políticas educacionales. En I Jornadas Latinoamericanas de estudios epistemológicos en Política Educativa organizadas por RELEPE.

Larrañaga O. (2014) Educación y superación de la pobreza en América. Un estudio del Proyecto "Mitigación de la Pobreza y Desarrollo Social". PNUD

OEI (2010) Metas Educativas 2021. Documento Final.

Moglia P. (2012) Epílogo. En Patricia Moglia y Carolina Cuesta (comp) en Las Didácticas Específicas y la formación del docente como profesional. San Martin: UNSAM Edita

Narodowsky M. (2016) Otro parche al sistema de puntaje docente. Disponible en el sitio web http://ar.bastiondigital.com/

Programa de las Naciones Unidas para el Desarrollo (PNuD). Disponible en el sitio web comunidad.fuac.edu.co/download/AREAS/2esx.pdf

Resoluciones del Consejo Federal de Educación No 83 y 94 (2009)

Romero C. (2009) Claves para mejorar la escuela secundaria. La gestión, la enseñanza y los nuevos actores. Buenos Aires: Noveduc Ediciones

Sen, A. (1992). Inequality reexamined, Cambridge: Harvard University Press.

Tenti Fanfani E. (2005) La condición docente. Análisis comparado de la Argentina, Brasil, Perú y Uruguay. Buenos Aires: Siglo XXI Editores

Terigi F. (2012) La enseñanza como problema en la formación en el ejercicio profesional. En Alejandra Birgin (comp.) Más allá de la capacitación. Debates acerca de la formación de los docentes en ejercicio. Buenos Aires: Paidós.

UNESCO-Instituto de Estadística (2013) Informe de Seguimiento de la Educación para Todos en el Mundo. Disponible en el sitio web www.unesco.org/new/es/education/themes/planningand-managing

Valsiner, J. (2001). Process structure of semiotic mediation in human development. Human Development, 44, 84-97. 
Desarrollo SOCIAL, POLÍtiCAS PÚBliCAS y CARRERA DOCENTE EN LA REPÚBLICA ARGENTINA. PRESENTE Y FUTURO DE LA FORMACIÓN DOCENTE CONTINUA. Daniel Ernesto Stigliano

DOI: http://dx.doi.org/10.15366/jospoe2018.8 Journal of Supranational POLICIES Of EDUCATION nº, pp. 37-50

Ziegler, S (2001) Clases del seminario de Postgrado "La educación secundaria: principales temas y problemas en perspectiva latinoamericana” FLACSO. Buenos Aires. 


\section{SOBRE EL AUTOR}

\section{Daniel Ernesto Stigliano}

Profesor en Química (Universidad Tecnológica Nacional). Licenciado en Educación (Universidad Nacional de Quilmes). Magister en Psicología Cognitiva y Aprendizaje (Facultad Latinoamericana de Ciencias Sociales). Master en Psicología Cognitiva (Universidad Autónoma de Madrid). Doctorando en Ciencias de la Educación (Universidad Nacional de La Plata). Consejero Académico de la Especialización en Docencia Universitaria de la Universidad Nacional del Oeste. Profesor de la Especialización en Docencia Universitaria de la Universidad de Buenos Aires. Supervisor Escolar. Asesor Pedagógico de la Fundación Pontificia Scholas Occurrentes de la Santa Sede. Miembro Honorario de la Asociación Mexicana de Metodología de la Ciencia y la Investigación. Ocupó cargos de gestión en escuelas e institutos de formación docente. Autor de nueve libros y numerosos artículos especializados en didáctica general y didáctica de las ciencias de perspectiva cognitivista, inclusión educativa y políticas para la diversidad.

Contact information: daniel_stigliano@yahoo.com.ar

Universidad de Buenos Aires 